\title{
Median Relative Partial Income Polarization Indices: Investigating Economic Polarization in Poland During the Years 2005-2015
}

\author{
Tomasz Panek ${ }^{1}$. Jan Zwierzchowski ${ }^{1}$ (D)
}

Accepted: 13 January 2020 / Published online: 21 January 2020

(c) The Author(s) 2020

\begin{abstract}
In recent years, more and more attention has been focused on the effects of economic growth and inequality changes on income polarization, as well as on the changes in the middle income class fraction. A significant part of the literature that deals with these issues is focused on polarization indices. However, the polarization indices proposed by researchers do not allow for an assessment of impact of the income distribution changes on the disappearance of the middle income class. Moreover, the general income polarization indices do not allow for assessment of polarization within the distinguished income classes. This study proposes a class of median relative polarization partial indices, which allows for a comprehensive assessment of the median relative polarization over time, within the distinguished income classes, as well as the impact of income distribution changes (its polarization or convergence) on the change of the middle income class fraction (its disappearance or increase). Using Social Diagnosis panel data (a study carried out by the Social Monitoring Council), the proposed new tool has been used to verify the hypothesis of whether changes in the household income distribution in Poland during the years 2005-2015 have led to income polarization within the three distinguished income groups-lower, middle and upper income classes. Empirical analysis shows that despite the lack of overall polarization of incomes in the household population, there was a convergence of incomes in the upper and lower income classes and polarization of incomes within the middle income class. It implies that the income distribution has not been petrified, and as on average individuals in the lower and upper income classes tend to reduce the distance to the median income, whereas the members of the middle income class tend to be pushed out of the middle class. Moreover, the flows of households into the middle income class were higher than the outflows from this class, resulting in economic convergence, i.e. changes in income distribution leading to an increase of the middle income class fraction.
\end{abstract}

Keywords Income polarization · Income inequality $\cdot$ Polarization indices

Jan Zwierzchowski

jan.zwierzchowski@sgh.waw.pl

Tomasz Panek

tompa10@interia.pl

1 Institute of Statistics and Demography, Warsaw School of Economics, A1. Niepodległości 162, 02-554 Warsaw, Poland 


\section{Introduction}

In the field of economics, the concept of economic polarization has been used since the eighties of the last century to describe the disappearance of the middle income class in the three class income distribution in the United States. The inspiration for scientific research on this process resulted from press reports on disappearance of the middle class in the country (Kuttner 1983; Thurow 1984). Verification of the reports conducted on the basis of empirical income distributions has confirmed a decline in the proportion of the middle income class (Rosenthal 1985; Horrigan and Haugen 1988). If this process were to be continued, it would eventually lead to a complete disappearance of the middle income class and to the formation of a bipolar income distribution.

The term "middle class" is commonly used, but vague, and while it is difficult to precisely define and measure a middle class for research purposes, it is possible to do so with a group in the middle of the income spectrum. Recording such measurements over a number of years allows to establish whether the proportion of people in the middle-income group is increasing or decreasing.

In this paper, we are focused solely on, what is called by Esteban and Ray (1994) a bipolarization. We understand polarization as the process by which the affected distribution becomes bi-polar. Moreover, in order to avoid ambiguity, the process of polarization leading to the disappearance of the middle income class is distinguished from the process of polarization that does not result in the disappearing of the middle income class. The first one is called the process of economic polarization and the other one is defined as the process of bi-polarization.

The notion of economic polarization is frequently used to describe the processes of changes in income distribution, not necessarily leading to the disappearance of the middle income class (Kot 2008). Some researchers believe that the process of economic polarization occurs when the poor get poorer and the rich get richer. However, this process does not necessarily have to result in the disappearance of the middle income class. In some cases, people from the middle income class may become affluent enough to become members of the "rich" class, while other may become impoverished enough for their income to be the same as that of the "poor" class. This type of bi-polarization may include the process of economic polarization, understood as the disappearance of the middle income class, as well as the process of making people from the "rich" class richer, and the poor from the "poor" class even more impoverished.

Economic polarization is often confused with the notion of income inequality. Inequality in income distribution is connected to the overall dispersion of distribution. It is related to the deviation of income distribution from the egalitarian distribution, whereas economic polarization describes a process in which income focuses on two separate poles or groups (one rich, and the other poor) which at the same time causes the disappearance of the middle income class (Rodriguez and Salas 2003). Moreover, as shown by Anderson (2004) in his study, an increase in polarization does not always lead to escalated income inequality, and similarly, an increase in income inequality does not necessarily results in the development of income polarization. An increase in polarization often leads to increased inequality, but the transition of those in the middle income class to the "poor" or "rich" income classes may also not cause an increase in inequality, assuming that changes may occur in the income of people in these classes. On the other hand, an increase in inequality can be a result of the enrichment of the "rich" or the impoverishment of the "poor" and, thus, may not necessarily affect the decrease of the middle income class. 
However, it should be noted that both the increase in income inequality and the increase in economic polarization usually coexist and both have a negative impact on economic development and social relations, although not necessarily of the same type. A large, developing middle class is what every nation strives towards. As proven by numerous studies (Banerjee and Duflo 2008), the middle class is vital for economic growth. The disappearance of the middle class is one of the main factors slowing down economic growth (see e.g., Banerjee and Duflo 2008; Kharas and Gertz 2010). A declining middle class also reduces equality of opportunities which results in a weakening of social cohesion and trust in economic and political institutions. Some economists claim that inequality is beneficial overall for stimulating growth, improves the quality of life for all members of the society and is a necessary part of social progress. Other economists conclude that inequality exploits disadvantaged population, hinders economic growth and hampers poverty reduction. Income inequality and the growing income gap between the rich and the poor also increase social conflicts and feeling of injustice (Keefer and Knack 2002; Duclos et al. 2004; Esteban and Ray 2011).

One of the basic approaches to measuring income polarization is the parametric approach, which requires quantitative measures called polarization indices to be used to assess income polarization changes (Kot 2008; Panek 2017). The most significant contribution to the quantitative analysis of the income polarization process was made by Esteban and Ray (1994) and Wolfson (1994). A set of summary measures of polarization was also proposed by Morris et al. (1994). These approaches to assessing and measuring income polarization were further developed by their authors and other researchers (Wolfson 1997; Esteban et al. 1998; Handcock and Morris 1999; Anderson 2004; Duclos et al. 2004; Montalvo and Reynal-Querol 2005; Esteban et al. 2007; Foster and Wolfson 2009; Lasso de la Vega et al. 2010; Esteban and Ray 2011). The polarization indices proposed by these researchers do not, however, allow to assess the degree of economic polarization in the strict sense i.e., the polarization that leads to the disappearance of the middle income class. Moreover, these indices do not allow for the assessment of polarization within distinguished income classes.

This paper attempts to make two contributions. First, we propose a class of median relative polarization partial indices based on the Morris et al. (1994) index. We chose this formula, as it is a dynamic measure, explicitly aimed at measuring polarization over time in a single population, as opposed to other measures, which are aimed at measuring polarization in distinct populations for comparison purposes. In the original paper authors proposed a general formula for median relative polarization index and its decomposition, providing additionally two indices aimed at measuring median relative polarization among individuals below and above median. In this study we further generalize, so that it is possible to measure median relative polarization in any given subgroup of the general population. These indices measure sign and strength of the median relative polarization within distinguished subgroups of the general population.

Moreover, we propose specific polarization indices to be used with panel databases, which allows for tracking the polarization of drawn individuals over time. Therefore, we can assess not only how the incomes have polarized in any given class, but also how incomes of individuals, who have belonged to any given subgroups in certain moments, have polarized over time. The proposed indices allow for analyzing the impact of changes inside the middle, lower and higher income classes on the incomes' distribution changes in the entire population (its polarization or convergence). We believe that the proposed measures tailored at panel data form a bridge between polarization and 
mobility measures. They offer further insight into analysis of income polarization and social class mobility as compared to the analysis tools that have been used so far.

The second contribution of the paper is to verify whether the hypothesis which assumes that changes in the household income distribution in Poland during the years 2005-2015 have led to changes in income distributions inside and outside the middle income class proves to be true.

Section 2 of the paper introduces the median relative polarization index (MRP) proposed by Morris, Bernhardt and Handcock. The class of the MRP partial indices, which allows to assess whether changes in income distribution lead to polarization inside the distinguished income classes is presented in Sect. 3. Moreover, the method of income mobility analysis applied in the study was presented. The statistical data used in this paper and the means of middle income class identification are discussed in Sects. 4.1 and 4.2. Section 4.3 reports and discusses empirical results, which have been concluded in Sect. 5 .

\section{The Median Relative Polarization Index}

The median relative polarization index (MRP) proposed by Morris et al. (1994) is based on a comparison of income distributions in two periods. Its objective is to study the differences between income distributions in reference and comparison populations (comparison and reference years). For this purpose, 'relative distribution' has been defined as the ratio of income density in the comparison year to the income density in the reference year evaluated at each quantile of the reference period. In order to isolate changes in the location of income distributions in the compared periods, distributions should initially be median matched by dividing the income distribution in the compared period by the growth rate of the income distribution median in the analyzed period:

$$
M e_{t=1, t=0}(y)=\frac{M e_{t=1}(y)}{M e_{t=0}(y)}, y \geq 0
$$

where $M e_{t=1}(y), M e_{t=0}(y)$ income distribution median in the comparison year $t=1$ and the reference year $t=0$.

The distribution of income can be broken into number of quantiles equal to the number of observations. If we denote by $r$ the $r$-th quantile rank that the income value $y$ has in the reference year, then the relative income distribution for the comparison year defines the following density function (Morris et al. 1994):

$$
g\left(y_{r}\right)=\frac{f_{t=1}\left(y_{r}\right)}{f_{t=0}\left(y_{r}\right)}, y_{r} \geq 0
$$

where $f_{t=1}\left(y_{r}\right), f_{t=0}\left(y_{r}\right)$ density functions of the median adjusted income for the comparison year $t=1$ and the reference year $t=0$ respectively.

Relative income distribution $g_{t=1}\left(y_{r}\right)$ is represented by the ratio of the density function at the $y$ income level in the comparison year to the density function at the same level of income in the reference year. The relative distribution is invariant to the scale of the distributions (up to a monotonic transformation).

Morris et al. proposed the following general form of the relative median polarization index (hereafter: MRP index), summing up all the changes in the relative distribution of income in the analyzed period: 


$$
M R P=4 \int_{0}^{1}\left|r-\frac{1}{2}\right| g\left(y_{r}\right) d r-1
$$

The median relative polarization index is based on changes in the shape of the income distribution in order to take account for polarization. The above index measures the average absolute deviation from the median of the effect function normalized to vary between -1 and 1 . The relative income distribution density of the studied population at the $y$ income level in comparison year $t=1$ is weighted by absolute differences between the reference quantiles rank of $y$ and the median $\left|r-\frac{1}{2}\right|$. Thus, the weight of relative density increases with the shifting of quantiles to the tails of income distribution. From a technical point of view, the integral in formula (3) is the average deviation of the relative distribution of income from the uniform distribution. The constants in the formula (3) scale index so it takes values in the range $[-1 ; 1]$. Moreover, the index value of 0 indicates a lack of polarization/convergence in the distribution of income during the analyzed period. Positive values of the index indicate income polarization, while negative values show a convergence of income distribution.

For individual data (discrete distribution), the MRP index takes the following form:

$$
M R P=\frac{4}{n_{t=1}} \sum_{i=1}^{n_{t=1}}\left|R_{i}-\frac{1}{2}\right|-1=\frac{4}{n_{t=1}} \sum_{i=1}^{n_{t=1}}\left|F_{t=0}\left(y_{i}\right)-\frac{1}{2}\right|-1
$$

where $R_{i}$ the fraction of units in the reference year for which income is lesser than that of the $i$-th unit from the comparison year i.e., the quantile rank of the relative income distribution of the surveyed units for the $i$-th income value.

\section{The MRP Partial Indices}

To assess whether changes in the distribution of income lead to changes in distribution inside and outside the middle class, we proposed to use the MRP partial indices, which are based on the MRP index. Within the framework of evaluating the process of changes in relative income distribution, they allow to assess whether median relative polarization or convergence occurred both within the distinguished classes, as well as among individuals who changed class belongingness in the analyzed period. In other words, the proposed indices answer the question whether the individuals in a given sub-population, on average, have come closer to the median income or rather have moved away from the median income over time. Moreover, their values indicate how strong this median relative polarization or convergence was. Therefore, they allow for a comparison of average, median relative polarization in various groups. However, due to their strongly non-linear nature, these indices should not be considered as a decomposition of the general formula.

\subsection{General Formula of MRP Indices for Sub-groups}

The MRP partial indices measure the impact on the surveyed unit income distribution changes on the median relative income polarization/convergence within various unit sub-groups. Let's consider a $\mathrm{G}$ group of individuals defined as all individuals, whose 
income in the current period belongs to an interval $\left[y_{\min }^{G} ; y_{\max }^{G}\right]$. The general form of the MRP partial index for the group $\mathrm{G}$ is defined as follows:

$$
M R P^{G}=\left(\frac{1}{D^{G}} \int_{F_{t=1}\left(y_{\min }^{G}\right)}^{F_{t=1}\left(y_{\max }^{G}\right)}\left|r^{G}-\frac{1}{2}\right| g\left(y_{r}\right) d r^{G}\right)^{\log \frac{1}{2 D^{G}} 2}-1,
$$

where

$$
D^{G}=\int_{F_{t=1}\left(y_{\min }^{G}\right)}^{F_{t=1}\left(y_{\max }^{G}\right)}\left|F_{t=1}\left(y^{G}\right)-\frac{1}{2}\right| d F_{t=1}\left(y^{G}\right),
$$

$r^{G}$ quantile ranks of units belonging to the $G$-th group in the reference year, $y^{G}$ incomes of units belonging to the $G$-th group, $y_{\min }^{G}, y_{\max }^{G}$ the lower and upper limit of incomes of individuals belonging to a given income sub-group $\mathrm{G}$.

The $D^{G}$ is a normalization coefficient and is equal to the average of the absolute differences between the comparison quantile rank of units belonging to the $G$-th group, in the income distribution of all of the units in the analyzed period, and the median. It is used for normalization purposes only. The complicated and non-linear nature of the formula (5) is caused by the fact that it needs to be normalized in three points, namely perfect convergence, perfect polarization and no change in incomes' distribution.

If there is virtually no change in the distribution of incomes over time, then the $D^{G}$ is equal to the current mean distance between the distribution function of incomes and 0.5 . In this case the whole formula (5), after deducting one, reduces to zero. However, if in the current period all individuals have equal income, then their distance of distribution function to 0.5 equals zero and the formula indicates -1 , which indicates a perfect convergence. Therefore, the formula is normalized in -1 and 0 (perfect convergence and no polarization/convergence scenarios) by its very nature.

However, if we observe perfect bi-polarization, an additional normalization factor is required. Let's assume that in the current period we observe a two-point distribution. The value of the mean distance of distribution function to 0.5 would be equal 0.5 , as all individuals have distribution functions of incomes equal to 0 or 1 . Therefore, the formula would be equal to:

$$
\left(\frac{1}{2 D^{G}}\right)^{\log \frac{1}{2 D^{G}} 2}-1=1
$$

which is equal to 1 as a result of raising to a power $\log _{\frac{1}{2 D^{G}}} 2$.

In the case of individual data, this index takes the form:

$$
M R P^{G}=\left(\frac{1}{D^{G} n_{t=1}^{G}} \sum_{i=1}^{n_{t=1}^{G}}\left|R_{i}-\frac{1}{2}\right|\right)^{\log \frac{1}{2 D^{G}} 2}-1,
$$

where: 


$$
D^{G}=\frac{\sum_{i=1}^{n_{t=1}^{G}}\left|F_{t=1}\left(y_{i, t=1}\right)-\frac{1}{2}\right|}{n_{t=1}^{G}} .
$$

Alternatively, the index (8) can be written as:

$$
M R P^{G}=\left(\frac{\sum_{i=1}^{n_{t=1}^{G}}\left|R_{i}-\frac{1}{2}\right|}{\sum_{i=1}^{n_{t=1}^{G}}\left|F_{t=1}\left(y_{i, t=1}\right)-\frac{1}{2}\right|}\right)^{\log \frac{1}{2 D^{G}} 2}-1 .
$$

The MRP partial index (8) is similar to the general MRP index (4) proposed by Morris et al., normalized in the range $[-1 ;+1]$. Moreover, the general index (4) may be considered as a unique case of the index (8) in which $G$ covers the entire surveyed population. If the $G$ group includes all units, the following relation shall apply:

$$
\sum_{i=1}^{n_{t=1}^{G}}\left|F_{t=1}\left(y_{i ; t=1}\right)-\frac{1}{2}\right|=\frac{1}{4} n_{t=1},
$$

and

$$
\log _{\frac{1}{2 D^{G}}} 2=\log _{\frac{1}{\frac{1}{4} * 2}} 2=1
$$

and the formula (8) comes down to the Eq. (4).

The literature concerning polarization provides a number of axioms' sets. Esteban and Ray (1991, 1994), and Duclos et al. (2004) proposed a set of axioms for general polarization measures, where polarization is understood as a tendency of a distribution to concentrate around a certain number of points. On the other hand, Foster and Wolfson (1992, 2009), Wolfson (1994), Wang and Tsui (2000) Bossert and Schworm (2008) and Chakravarty and D'Ambrosio (2010) provided axioms for bi-polarization indices, where polarization is considered to result in a distribution concentrated around two points at its tails. As measures proposed in the study are specifically designed to measure bi-polarization, that is median relative polarization, we will consider their properties in the context of the latter set of axioms.

Firstly, we will consider a Within Group Clustering axiom also known as NonDecreasing Spread. This axiom requires that a movement of incomes from the middle position to the tails of the income distribution results in the relevant measure showing at least as much polarization as before. This axiom is also referred to as a transfer axiom, as it covers the notion that a Pigou-Dalton transfer type in the lower half of the income distribution should not lead to the decrease in polarization.

The second axiom is known as Between Group Spread or Non-Decreasing Bipolarity. It requires that a clustering of incomes below or above median does not lead to a decrease in polarization measure values.

The third considered axiom is a Scale Invariance axiom, which requires that multiplying income values by a constant does not lead to changes in polarization measure values.

Axiom 1 (Within group clustering) Consider two populations with income distributions A and B with the same median $y_{m e}=y_{m e}^{A}=y_{m e}^{B}$, the same number of observations $n$, 
consisting of individuals ordered by their income: $\left(y_{1}^{A} ; y_{2}^{A} ; \ldots ; y_{n}^{A}\right)$ and $\left(y_{1}^{B} ; y_{2}^{B} ; \ldots ; y_{n}^{B}\right)$. Moreover, one of the following holds:

(a) $\forall_{i=1 ; \ldots ; m e} y_{i}^{A}=y_{i}^{B}$ and $\exists_{i>m e} y_{i}^{A}>y_{i}^{B}$;

(b) $\forall_{i=m e ; \ldots ; n} y_{i}^{A}=y_{i}^{B}$ and $\exists_{i>m e} y_{i}^{A}<y_{i}^{B}$;

(c) $\exists_{i>m e} y_{i}^{A}>y_{i}^{B}$ and $\exists_{i>m e} y_{i}^{A}<y_{i}^{B}$

Then $\mathrm{A}$ is at least as polarized as $\mathrm{B}$.

Axiom 2 (Between group spread) Consider two populations with income distributions A and B with the same median $y_{m e}=y_{m e}^{A}=y_{m e}^{B}$, the same number of observations $n$, consisting of individuals ordered by their income: $\left(y_{1}^{A} ; y_{2}^{A} ; \ldots ; y_{n}^{A}\right)$ and $\left(y_{1}^{B} ; y_{2}^{B} ; \ldots ; y_{n}^{B}\right)$. If $\forall_{i=1 ; \ldots ; m e} y_{i}^{A} \leq y_{i}^{B}$ and $\forall_{i=m e ; \ldots ; n} y_{i}^{A} \geq y_{i}^{B}$ then $\mathrm{A}$ is at least as polarized as $\mathrm{B}$.

Axiom 3 Let the distributions A and B have the same median income Me. Then if $\mathrm{B}$ is more polarized than A, so is B' relative to A', where B' and A' are obtained from B and A respectively by dividing all incomes by their median Me.

Proposition 1 Let $A$ be the distribution function of incomes in the current period and $B$ the distribution function of incomes in the reference period. The proposed index $M R P^{G}$ calculated for any group taken from distribution $A$ and compared to distribution $B$ satisfies Axiom 1.

Proof 1 If the conditions listed in the Axiom 1 are met then $F_{t=1}\left(y_{i}^{A}\right) \leq F_{t=0}\left(y_{i}^{A}\right)=r_{i}$ for $i$ such that $y_{i}^{A}<y_{m e}$ and $F_{t=1}\left(y_{i}^{A}\right) \geq F_{t=0}\left(y_{i}^{A}\right)=r_{i}$ for $i$ such that $y_{i}^{A}>y_{m e}$. Therefore, in both cases:

$$
\left|F_{t=1}\left(y_{i}^{A}\right)-\frac{1}{2}\right| \leq\left|F_{t=0}\left(y_{i}^{A}\right)-\frac{1}{2}\right|=\left|r_{i}-\frac{1}{2}\right|
$$

That is the distance to the $\frac{1}{2}$ of the distribution function of any given income in the current period incomes distribution is no greater than the same distance of the distribution from the reference period.

A similar relation holds for the aggregated values of distribution functions over any subgroup G:

$$
\int_{F_{t=1}\left(y_{\min }^{G}\right)}^{F_{t=1}\left(y_{\max }^{G}\right)}\left|r^{G}-\frac{1}{2}\right| g\left(y^{G}\right) d r^{G} \geq \int_{F_{t=1}\left(y_{\min }^{G}\right)}^{F_{t=1}\left(y_{\max }^{G}\right)}\left|F_{t=1}\left(y^{G}\right)-\frac{1}{2}\right| d F_{t=1}\left(y^{G}\right)=D^{G}
$$

Thus:

$$
\frac{1}{D^{G}} \int_{F_{t=1}\left(y_{\min }^{G}\right)}^{F_{t=1}\left(y_{\max }^{G}\right)}\left|r^{G}-\frac{1}{2}\right| g\left(y^{G}\right) d r^{G} \geq 1
$$

And: 


$$
M R P^{G}=\left(\frac{1}{D^{G}} \int_{F_{t=1}\left(y_{\min }^{G}\right)}^{F_{t=1}\left(y_{\max }^{G}\right)}\left|r^{G}-\frac{1}{2}\right| g\left(y^{G}\right) d r^{G}\right)^{\log \frac{1}{2 D^{G}} 2}-1 \geq 0 .
$$

Proposition 2 Let $A$ be the distribution function of incomes in the current period and $B$ the distribution function of incomes in the reference period. The formula (5) calculated for group $G$ fulfills Axiom 2.

Proof 2 For the individuals below median we know that $\forall_{i=1 ; \ldots ; m e} y_{i}^{A} \leq y_{i}^{B}$, therefore, the distribution function for any income level below median in the current period is not larger than the distribution function for the same value in the reference period:

$$
\forall_{i=1 ; \ldots ; m e} F_{t=1}\left(y_{i}^{A}\right) \geq F_{t=0}\left(y_{i}^{A}\right)=r_{i},
$$

Because medians are equal in both periods (distributions are median matched) we have the following: $\forall_{i=1 ; \ldots ; m e} F_{t=1}\left(y_{i}^{A}\right)<\frac{1}{2}$ and $\forall_{i=1 ; \ldots ; m e} F_{t=0}\left(y_{i}^{A}\right)<\frac{1}{2}$,

Therefore:

$$
\forall_{i=1 ; \ldots ; m e}\left|F_{t=1}\left(y_{i}^{A}\right)-\frac{1}{2}\right| \leq\left|F_{t=0}\left(y_{i}^{A}\right)-\frac{1}{2}\right|=\left|r_{i}-\frac{1}{2}\right|
$$

Similarly, for individuals above median we know that $\forall_{i=m e ; \ldots ; n} y_{i}^{A} \geq y_{i}^{B}$. Thus:

$$
\forall_{i=m e ; \ldots ; n} F_{t=1}\left(y_{i}^{A}\right) \leq F_{t=0}\left(y_{i}^{A}\right)=r_{i}
$$

And as $\forall_{i=1 ; \ldots ; m e} F_{t=1}\left(y_{i}^{A}\right)>\frac{1}{2}$ and $\forall_{i=1 ; \ldots ; m e} F_{t=0}\left(y_{i}^{A}\right)>\frac{1}{2}$, we have:

$$
\forall_{i=m e ; \ldots ; n}\left|F_{t=1}\left(y_{i}^{A}\right)-\frac{1}{2}\right| \leq\left|F_{t=0}\left(y_{i}^{A}\right)-\frac{1}{2}\right|=\left|r_{i}-\frac{1}{2}\right|
$$

The rest of the proof follows the same route as Proof 1 (compare Eq. 13), and therefore, $M R P^{G} \geq 0$.

Proposition 3 The proposed index $M R P^{G}$ fulfills Axiom 3

Proof 3 The proposed index is solely based on the relative distribution of incomes. It utilizes distribution functions of incomes, rather than incomes themselves. As any uniform scaling does not alter values of distribution functions for individuals, it will also not affect the values of the indices for any subgroup. Moreover, the income distributions are median matched, meaning that the current incomes are divided by the growth rate of the income distribution median in the analyzed period. Therefore, multiplying incomes in one or both periods by a constant does not affect the values of $M R P^{G}$ 


\subsection{MRP Partial Indices for Panel Data}

Panel data are better suited for studying the changes of processes and phenomena in time as compared to cross-sectional data. In the case of cross-sectional data, changes in the value of polarization indices over time may not only result from the changes in the distribution of household incomes, but also be caused by sampling error, as different samples of households are analyzed in the two compared periods. Moreover, the panel data allow for tracking units of observation over time and assessing their polarization at an individual level.

The second set of the MRP partial indices, tailored for use with panel datasets in empirical research, allows for a deeper analysis of changes in income distribution. In this approach the analyzed subpopulations are defined on the basis of their belongingness to certain income groups in two distinct time moments. For individual data, the general form of this type of the MRP partial index is:

$$
M R P_{P}^{G}=\left(\frac{1}{D^{G} n^{G}} \sum_{i=1}^{n^{G}}\left|R_{i}-\frac{1}{2}\right|\right)^{\log \frac{1}{2 D^{G}}} 2-1++\frac{2}{n^{G}}\left(\sum_{i=1}^{n^{G}}\left(\left|F_{t=1}\left(y_{i, t=1}\right)-\frac{1}{2}\right|-\left|F_{t=0}\left(y_{i, t=0}\right)-\frac{1}{2}\right|\right)\right),
$$

where $n^{G}$ is the number of individuals belonging to a given group $\mathrm{G}$, identified on the basis of their belongingness to certain groups in both periods.

The first element of the index $M R P_{P}^{G}$ is identical to the proposed $M R P^{G}$ index for empirical data [defined by formula (5)] and it takes into account the degree of change in income polarization (convergence) of the considered class of units in relation to the whole income distribution over time. However, in order to assess polarization in a specific group of individuals, it is insufficient. Let us consider a population of three equal groups (lower, middle and upper classes) whose members have equal incomes of 1,2 and 3 respectively at time 0 . At time 1, all individuals who previously had incomes 1 shift to the middle income class and now experience income of 2 . Vice versa, all individuals from the middle class drop to the lower income class and have income of 1 . As a consequence, general distribution of incomes does not change, and the index defined by the formula (5) will be equal to 0 . However, the individuals constituting the lower and middle income classes at time 1 have experienced respectively very strong convergence and polarization in the analyzed period.

Therefore, we propose to add a second element to the formula for panel data:

$$
\frac{2}{n^{G}}\left(\sum_{i=1}^{n^{G}}\left(\left|F_{t=1}\left(y_{i, t=1}\right)-\frac{1}{2}\right|-\left|F_{t=0}\left(y_{i, t=0}\right)-\frac{1}{2}\right|\right)\right)
$$

which will track individual shifts in relative income distribution. Basically, it compares the distance between individuals' income distribution function and the median income in the two analyzed periods. It takes positive values when on average individuals within a certain group shift away from the median income and negative values in an opposite situation.

This added element (22) is also normalized in the $[-1 ; 1]$ interval. If all individuals in a given group move from the median income in the reference period to minimum or maximum income in the analyzed period, their average difference in distances from median will be equal to 0.5 and the whole element defined by (22) will be equal to 1 and will indicate perfect polarization. In the opposite case it will be equal to -1 and will indicate perfect convergence. If nothing changes over time it will be equal to 0 . 
When the analyzed group consists of the entire population of units, the element defined by (22) takes the value 0 and the $M R P_{P}^{G}$ index comes down to the general MRP index, as proposed by Morris et al. (1994). Therefore, the $M R P_{P}^{G}$ partial index should be seen as a further generalization of the MRP index formula (5). Moreover, it differs from the original index if and only if it is calculated for a subgroup.

The $M R P_{P}^{G}$ does not in general satisfiy axioms 1-2. The first part of formula (21) is an empirical form of formula (5) and it points to non-negative polarization whenever the assumptions of axioms 1-2 are met. However, the second part of (21), given by formula (22) may take positive or negative values, depending on the average change in the incomes of individuals who belong to the analyzed sub-group.

The reason for which the proposed index fails to satisfy the two axioms is the fact that these axioms were created for comparing measures assessing only changes in the distribution as a whole. In other words these axioms implicitly follow a notion that switching incomes between individuals should not be reflected by the polarization measures and only a shape of the distribution should be considered while assessing polarization.

The proposed index (21) additionally assess the polarization/convergence experienced at an individual level. In the proposed approach, we make an attempt at tracing individuals' incomes, which may be considered as an analysis of mobility rather than polarization. However, in fact the observed polarization at aggregated level is caused by the mobility of individuals. Therefore, we believe that the proposed approach bridges the two types of analysis of incomes. Moreover, it may facilitate identification of causes for the observed polarization/convergence due to specific changes in income distribution in certain population subgroups.

In the context of analyzing economic polarization, we propose to calculate values of index $M R P_{P}^{G}$ for individuals who move from one income class to another as well as for those who maintain their class belongingness over time. Firstly, we define the $M R P_{P}^{E P}$ index associated with individuals who experience economic polarization, namely who have entered or exited the middle income class over the analyzed period:

$$
\begin{aligned}
\operatorname{MRP}_{P}^{E P}= & \left(\frac{1}{D^{E P} n^{E P}} \sum_{i=1}^{n^{E P}}\left|R_{i}-\frac{1}{2}\right|\right)^{\log \frac{1}{2 D^{E P}}} 2 \\
& +\frac{2}{n^{E P}}\left(\sum_{i=1}^{n^{E P}}\left(\left|F_{t=1}\left(y_{i, t=1}\right)-\frac{1}{2}\right|-\left|F_{t=0}\left(y_{i, t=0}\right)-\frac{1}{2}\right|\right),\right.
\end{aligned}
$$

where $n^{E P}=n^{M C \rightarrow}+n^{\rightarrow M C}$ the number of units that have changed their belonging to the given income class resulting in changes in the middle income fraction, $n^{M C \rightarrow}, n^{\rightarrow M C}$ the number of units that have accordingly left the middle income class and entered the middle income class during the evaluated period.

The $M R P_{P}^{E P}$ index is associated with economic polarization, i.e. taking into consideration income polarization leading to the decrease of the middle income class fraction $\left(M R P_{P}^{M C \rightarrow}\right)$, by moving units from this class to the lower or higher income class and the economic convergence giving rise to the increase of the middle income class fraction $\left(M R P_{P}^{\rightarrow M C}\right)$ due to the transition of class units from the lower or the higher income class.

Moreover, we can consider separate indices for the two groups of units. The first of the partial indices, measuring the income polarization resulting in the decrease of the 
middle income class fraction is associated with economic polarization and can be calculated as follows:

$$
\begin{aligned}
M R P_{P}^{M C \rightarrow}= & \left(\frac{1}{D^{M C \rightarrow} n^{M C \rightarrow}} \sum_{i=1}^{n^{M C} \rightarrow}\left|R_{i}-\frac{1}{2}\right|\right)^{\log \frac{1^{2 D^{M C} \rightarrow}}{2}}-1 \\
& +\frac{2}{n^{M C \rightarrow}}\left(\sum_{i=1}^{n^{M C} \rightarrow}\left(\left|F_{t=1}\left(y_{i, t=1}\right)-\frac{1}{2}\right|-\left|F_{t=0}\left(y_{i, t=0}\right)-\frac{1}{2}\right|\right)\right) .
\end{aligned}
$$

The second partial index, which should be associated with economic convergence, is calculated as follows:

$$
\begin{aligned}
M R P_{P}^{\rightarrow M C}= & \left.\left(\frac{1}{D^{\rightarrow M C} n^{\rightarrow M C}} \sum_{i=1}^{n^{\rightarrow M C}}\left|R_{i}-\frac{1}{2}\right|\right)\right|^{\log \frac{1}{2 D^{\rightarrow M C}}}-1 \\
& +\frac{2}{n^{\rightarrow M C}}\left(\sum_{i=1}^{n^{\rightarrow M C}}\left(\left|F_{t=1}\left(y_{i, t=1}\right)-\frac{1}{2}\right|-\left|F_{t=0}\left(y_{i, t=0}\right)-\frac{1}{2}\right|\right) .\right.
\end{aligned}
$$

Similarly, we may define the index $M R P_{P}^{N E P}$ measuring the income polarization (convergence) that does not change the middle income class fraction, which includes polarization (convergence) inside the middle income class and outside the middle income class. It takes the following form:

$$
\begin{aligned}
M R P_{P}^{N E P}= & \left(\frac{1}{D^{N E P} n^{N E P}} \sum_{i=1}^{n^{N E P}}\left|R_{i}-\frac{1}{2}\right|\right)^{\log \frac{1}{\frac{1}{D^{N E P}}} 2}-1 \\
& +\frac{2}{n^{N E P}}\left(\sum_{i=1}^{n^{N E P}}\left(\left|F_{t=1}\left(y_{i, t=1}\right)-\frac{1}{2}\right|-\left|F_{t=0}\left(y_{i, t=0}\right)-\frac{1}{2}\right|\right),\right.
\end{aligned}
$$

where $n^{N E P}=n^{M C}+n^{L C ; H C}$ the number of units belonging to the same income class in both periods, $n^{M C}, n^{L C ; H C}$ the numbers of units belonging accordingly to the middle income class and to the lower or the higher class during both considered periods.

The index measuring the income polarization of units belonging in both periods to the middle income class is calculated in the following way:

$$
\begin{aligned}
\operatorname{MRP}_{P}^{M C}= & \left.\left(\frac{1}{D^{M C} n^{M C}} \sum_{i=1}^{n^{M C}}\left|R_{i}-\frac{1}{2}\right|\right)^{\log \frac{1}{2 D^{M C}}}\right)^{2}-1 \\
& +\frac{2}{n^{M C}}\left(\sum_{i=1}^{n^{M C}}\left(\left|F_{t=1}\left(y_{i, t=1}\right)-\frac{1}{2}\right|-\left|F_{t=0}\left(y_{i, t=0}\right)-\frac{1}{2}\right|\right)\right)
\end{aligned}
$$

On the other hand, the index constituting the measure of income polarization (convergence) concerning units not belonging to the middle income class in both periods is calculated as follows: 


$$
\begin{aligned}
\operatorname{MRP}_{P}^{L C ; H C}= & \left(\frac{1}{D^{L C ; H C} n^{L C ; H C}} \sum_{i=1}^{n^{L C ; H C}}\left|R_{i}-\frac{1}{2}\right|\right)^{\log \frac{1}{2 D^{L C ; H C}}}{ }^{2}-1 \\
& +\frac{2}{n^{L C ; H C}}\left(\sum_{i=1}^{n^{L C ; H C}}\left(\left|F_{t=1}\left(y_{i, t=1}\right)-\frac{1}{2}\right|-\left|F_{t=0}\left(y_{i, t=0}\right)-\frac{1}{2}\right|\right),\right.
\end{aligned}
$$

where $n^{L C ; H C}$ the number of units belonging to the lower or the higher income class both in the reference year and in the comparison year.

\subsection{Analysis of Flows of Households Between Income Classes}

The analysis of the income distribution changes based on the proposed indices should be supplemented by the examination of the individuals' flows between income classes in the considered period.

For the evaluation of income mobility the Shorrocks index (1978), index often used in practice was applied:

$$
M^{S}=\frac{n-\operatorname{tr}(N)}{n-1}
$$

where $\operatorname{tr}(\mathbf{N})$ trace of the matrix of flows, that is the number of units which have not changed their income class belongingness during the analysed period.

Index (25) takes values in the range [0, 1]. The higher the value of the index, the greater the units' mobility. When decomposing index (29), and expanding its analytical capacities, we obtain the following:

$$
M^{S}=\frac{n-\operatorname{tr}(N)}{n-1}=\frac{n^{G \rightarrow G \uparrow}+n^{G \rightarrow G \downarrow}}{n-1}=\frac{n^{G \rightarrow G \uparrow}}{n-1}+\frac{n^{G \rightarrow G \downarrow}}{n-1}=M^{S+}+M^{S-}
$$

where $n^{G \rightarrow G \uparrow}$ number of units which flowed from the lower income group to the higher income group, $n^{G \rightarrow G \downarrow}$ number of units which flowed from the higher income group to the lower income group.

The first of the components on the right side of the equation indicates the percentage of units which moved to the higher income class between the two analysed periods. The second component of the sum is the percentage of units which moved to the lower income class in the studied period. As a complement for the Shorrocks mobility index (29), we may propose another decomposition. Formula (29) defines indices which measure relative flows inside and outside the middle income class:

$$
M^{S}=\frac{n^{\rightarrow M C}}{n-1}+\frac{n^{M C \rightarrow}}{n-1}+\frac{n^{L M \leftrightarrow H C}}{n-1}=M^{\rightarrow M C}+M^{M C \rightarrow}+M^{L M \leftrightarrow H C}=
$$

where $n^{\rightarrow M C}$ number of units that entered the middle income class, $n^{M C} \rightarrow$ number of units that left the middle income class, $n^{L M \leftrightarrow H C}$ number of units that shifted between the lower and higher income classes.

The values of indices $M^{\rightarrow M C}$ and $M^{M C \rightarrow}$ are related to the process of economic polarization, as they inform on the fractions of total population that have experienced economic convergence and economic polarization respectively, whereas the third element, $M^{L M \leftrightarrow H C}$ 
does not need to bear any information relevant from the point of view of the analysis of economic polarization.

\section{Empirical Example: Changes in the Relative Income Distribution in Poland During the Years 2005-2015}

\subsection{Data Characteristics}

The basis for the analysis of changes in relative income distributions in Poland in 2005-2015 is constituted by data from the Social Diagnosis research project conducted by the Council for Social Monitoring (Czapiński et al. 2015). The project is a panel study, where each subsequent wave involves all available households (which agreed to continue to participate in the survey) from previous rounds. All households that participated in the research project during both years, i.e. in 2005 and in 2015, were analyzed in the study. The Social Diagnosis survey is conducted using a representative method that allows to generalize, with a desired level of precision, the results obtained to the whole population of Poland.

The income category used is the net monthly equivalent income of households. The net monthly equivalent incomes were calculated by dividing monthly household net incomes by the equivalence scales. Monthly household incomes were expressed at constant 2015 prices (incomes from the 2005 year were adjusted with a relevant consumer price index).

The equivalence scales used in the survey were estimated on the basis of the procedure using data on the amount of expenditure of the households from household budget surveys (Szulc 2003; Panek 2015). The procedure was conducted using Household Budget Survey data. It takes into account the fact that households of different compositions spend income in different ways. For example, the households of young people spend less on medical care and more on food, unlike the households of older people. At the same time, it has been assumed that the consumption structure of households reflects their actual needs. Households of single employees between 30 and 59 years of age were established as the point of reference (as a standard household, with the equivalence scale of 1). The value of the equivalence scale for any other household may be then interpreted as the number of standard households it includes (in this case, the number of standard persons). The equivalence scales were estimated on the basis of the following formula:

$$
\ln \left(m_{i}\right)=\frac{1}{2} \sum_{j=1}^{m} \sum_{s=1}^{n}\left[m_{s j}\left(w_{s i}+w_{s r}\right)\right] \ln \left(\frac{\boldsymbol{A}_{j i}}{\boldsymbol{A}_{\boldsymbol{j} \boldsymbol{r}}}\right),
$$

where $m_{i}$ equivalence scale for the $i$-th household, $w_{s i} ; w_{s r}$ the percentage of expenditure of the $i$-th and $r$-th household on the $s$-th good or group of goods; in this case the $r$-th household is the standard household, $m_{s j}$ the elasticity of expenditure for the $s$-th good in relation to the $j$-th demographic characteristic $(j=1,2, \ldots, m), \boldsymbol{A}_{j i} ; \boldsymbol{A}_{j i}$ the vectors of demographic characteristics of the $i$-th and $r$-th household.

In the presented study, the vectors of demographic characteristics were based on the number of adult persons in the household (above 16 years of age), the number of children (below 10 years of age and from 10 to 15 years of age) and the age of the head of household (16-29 years, 30-60 years and more than 60). The $m_{s j}$ parameters were obtained through the estimation of the model of consumption demand, with the following explanatory 
variables: the expenditure of the household, the number of adult persons and children in the household and the prices of consumer goods. These are interpreted as the demographic elasticities of expenditure on specific goods. Thus, the equivalence scale obtained on the basis of Eq. (32) is a geometric mean of the elasticities of expenditure in relation to the demographic variables weighted with the shares of expenditure on specific goods in the total expenditure.

\subsection{The Identification of the Middle Income Class}

Determining whether changes in the distribution of household incomes are a cause of the disappearance of the middle income class requires a prior defining of the limits of this class, i.e. the determination of income interval for the middle income class $\left[\mathrm{y}^{\prime} ; \mathrm{y}^{\prime \prime}\right]$ and the calculation of the fraction of households in the middle income class. There are various definitions of the middle income class (Atkinson and Brandolini 2011). In our study, the limits of the middle income class $y_{i}$, were established at the level of $75 \%$ and $200 \%$ of the median monthly equivalent net income of households as proposed by Eurofound (VacasSoriano and Fernandez-Macias 2017). These boundaries equaled to 645 PLN and 2151 PLN, respectively.

\subsection{Is Poland's Middle Income Class Disappearing?}

Firstly, an analysis of changes in household income distribution in 2005-2015 was conducted using the general polarization index (4). In the surveyed period 2005-2015 there were no significant changes in the income distribution of households in Poland- the polarization index for the whole population virtually equals zero, as it assumed the value of -0.0006 (Table 1).

A lack of polarization/convergence in the distribution of households' incomes in 2005-2015 was accompanied by a decrease in the households' fraction in the lower (decrease from 17.2 to $15 \%$ ) and higher income classes (decrease from 8.7 to $7.8 \%$ ). At the same time, the fraction of households in the middle income group increased from 74.1 to $77.2 \%$, therefore an economic convergence was observed (compare Table 2).

In total, approximately $31 \%$ of households between 2005 and 2015 shifted between income classes (Tables 3,4). The number of households which have flowed to the higher income classes in the compared 2 years was slightly higher (16.2\% of households) than the number of those which moved to the lower income classes (15\% of households). Moreover, $13.5 \%$ of the total population has moved outside the middle income class, while $16.6 \%$ of the total population has moved to the middle income class from lower or higher classes in the analyzed period. These observations combined may suggest a convergence in the incomes distribution and seemingly contradict the value of the overall MRP index.

In total, polarization was observed inside the middle income class, as the estimated value of the MRP partial index equalled 0.0073. Within the higher and lower income classes the incomes have converged. Moreover, the observed convergence of incomes in these two classes was stronger than corresponding polarization in the middle class, as the MRP partial indices reached the values of -0.1110 and -0.0492 , respectively. The reason for which the overall value of the MRP index equalled zero is that the middle income class was significantly larger than the two outlying classes, which compensated for the lower 
Table 1 Median relative polarization indices for the distribution of net monthly equivalent income in Poland in 2005-2015

\begin{tabular}{lclrcc}
\hline Index type & MRP indices & Standard error & $\mathrm{z}$ statistic & $p$ value & $95 \%$ confidence interval \\
\hline$M R P$ & -0.0006 & 0.036 & -0.017 & 0.986 & {$[-0.069 ; 0.072]$} \\
$M R P^{M C}$ & 0.0073 & 0.039 & 0.187 & 0.852 & {$[-0.062 ; 0.083]$} \\
$M R P^{L C}$ & -0.0492 & 0.136 & -0.361 & 0.718 & {$[-0.329 ; 0.141]$} \\
$M R P^{H C}$ & -0.1110 & 0.190 & -0.583 & 0.560 & {$[-0.579 ; 0.801]$} \\
$M R P_{P}^{E P}$ & -0.0534 & 0.062 & -0.859 & 0.390 & {$[-0.167 ; 0.074]$} \\
$M R P_{P}^{M C \rightarrow}$ & $0.3351^{*}$ & 0.100 & 3.343 & 0.000 & {$[-0.141 ; 0.532]$} \\
$M R P_{P}^{\rightarrow M C}$ & $-0.3987 *$ & 0.047 & -8.456 & 0.000 & {$[-0.486 ;-0.301]$} \\
$M R P_{P}^{N E P}$ & 0.0232 & 0.033 & 0.704 & 0.481 & {$[-0.039 ; 0.090]$} \\
$M R P_{P}^{M C}$ & 0.0298 & 0.036 & 0.825 & 0.409 & {$[-0.039 ; 0.103]$} \\
$M R P_{P}^{L C ; H C}$ & -0.0625 & 0.109 & -0.575 & 0.565 & {$[-0.274 ; 0.145]$} \\
\hline
\end{tabular}

*Polarization partial indices are significantly higher/lower than 0 at the 0.05 . In the case of the $M R P_{P}^{M C \rightarrow}$ index, the alternative hypothesis is right-sided and in the case of the $M R P_{P}^{\rightarrow M C}$ index it is left-sided. To determine standard errors of estimation and confidence intervals, the bootstrap method was applied using 10,000 replications

Table 2 Income classes and average equivalent incomes of households in Poland in 20052015. Source: Own analysis based on Social Diagnosis data

\begin{tabular}{|c|c|c|c|c|c|c|c|c|}
\hline \multirow[t]{2}{*}{ Year } & \multicolumn{2}{|c|}{$\begin{array}{l}\text { Class limits } \\
\text { (in PLN) }\end{array}$} & \multicolumn{3}{|c|}{ Household fractions } & \multicolumn{3}{|c|}{$\begin{array}{l}\text { Average incomes (in } \\
\text { PLN) }\end{array}$} \\
\hline & $\mathrm{y}^{\prime}$ & $y^{\prime \prime}$ & $\mathrm{w}^{\mathrm{LC}}$ & $\mathrm{w}^{\mathrm{MC}}$ & $w^{\mathrm{HC}}$ & $\overline{y^{L C}}$ & $\overline{y^{M C}}$ & $\overline{y^{H C}}$ \\
\hline 2005 & 645 & 2.151 & 0.172 & 0.741 & 0.087 & 476 & 1.190 & 3.487 \\
\hline 2015 & 989 & 3.297 & 0.150 & 0.772 & 0.078 & 741 & 1.797 & 4.489 \\
\hline
\end{tabular}

value of the relevant indices in absolute terms. However, the stronger convergence in the tails, as opposed to the middle income class, was a reason for which the fraction of households in the middle income group increased.

That said, both the convergence of income distribution in the lower and upper income classes and the polarization in the middle class were accompanied with an increase in wealth. The average real incomes of households increased during the analyzed period by $29 \%$ in the lower, by $51 \%$ in the middle and by $56 \%$ in the upper income class (Table 2).

The second type of the MRP partial indices (formulas 23-27) allows for a direct analysis of polarization among households, which belonged to certain income classes in the two analyzed years. The households that transitioned from the middle income class to the lower or higher income class (13.5\% of the total population) in 2015 compared to 2005, experienced on average a strong polarization of incomes, as relevant index was equal to 0.33 (Table 1). Similarly, reverse transitions (16.6\% of the total population of households) resulted in the strong convergence of incomes, as the polarization index for this group equalled -0.4 . The power of economic polarization was slightly smaller than the power of economic convergence and, as a result, the MRP partial index associated with changes in the middle income class fraction reached -0.0534 . This value measures the sign and strength of economic polarization. It means that on average individuals entering middle income class experienced stronger shift in their relative incomes as compared to those exiting middle income class. 
As a result, the observed household transitions between income classes led to economic convergence, i.e. the convergence of income distribution, which at the same time resulted in an increase in the middle income class fraction.

The values of the MRP partial indices describing changes in income distribution which do not lead to changes in the middle income class fraction differed in the three groups. A polarization of income distribution was observed among households which belonged to the middle income class during both analyzed periods. The value of the MRP partial index measuring the power and direction of these changes equalled 0.0298. However, changes in household incomes which in both compared years remained outside the middle class caused a convergence of the income distribution in these income classes - the relevant index reached the value of -0.0625 . A joint analysis of changes in household income distribution, which did not affect changes in the fraction of households in the middle income class (and did not affect the economic polarization or convergence) indicates the polarization of this distribution in the analyzed years 2015 and 2005 and the value of the relevant partial index was equal to 0.0232 . The reason for which a polarization was observed in this aggregate is, once again, a higher fraction of those who were observed inside the middle income class in both years $(60.6 \%$ of the total population) as compared to the fraction of those who stayed outside the middle income class (9.5\% of the total population, compare Table 3). Therefore, the higher absolute value of the convergence index outside the middle class, as compared to the polarization index inside the middle class, was compensated by the larger number of individuals inside the middle class.

These observations suggest that the income structure in Poland is not petrified, as on average, households which stayed outside the middle income class converge towards median income, while households inside the middle income class were experiencing polarization in the analyzed period, which in time may force them outside of the middle income class. Moreover, as the relevant index outside the middle income class is stronger, as compared to the polarization index within the middle class, we may expect to observe further increase in the fraction of households inside the middle income class in the near future.

Table 3 Flows of household between income classes in Poland in 2005-2015
Table 4 Mobility indices for Poland in 2005-2015

\begin{tabular}{lllll}
\hline $\begin{array}{l}\text { Belonging to the given sub- } \\
\text { group of population in 2005 } \\
\text { (in \%) }\end{array}$ & $\begin{array}{l}\text { Belonging to the given } \\
\text { subgroup of population in } \\
\text { 2015 (in \%) }\end{array}$ & Total \\
\cline { 2 - 5 } & $\begin{array}{l}\text { i } G^{L C} \\
\text { i } i \in G^{M C}\end{array}$ & \\
\hline$i \in G^{L C}$ & 6.3 & 10.4 & 0.5 & 17.2 \\
$i \in G^{M C}$ & 8.3 & 60.6 & 5.2 & 74.1 \\
$i \in G^{H C}$ & 0.4 & 6.2 & 2.1 & 8.7 \\
Total & 15.0 & 77.2 & 7.8 & 100 \\
\hline
\end{tabular}

Type of mobility indices

Estimated indices' values (2015 to 2005)

\begin{tabular}{ll}
\hline $\mathrm{S}$ & 0.312 \\
$M^{S+}$ & 0.162 \\
$M^{S-}$ & 0.150 \\
$M^{\rightarrow M C}$ & 0.166 \\
$M^{M C \rightarrow}$ & 0.135 \\
$M^{L M \leftrightarrow H C}$ & 0.011 \\
\hline
\end{tabular}




\section{Conclusions}

This paper proposes a new class of MRP partial indices, which enable a deeper analysis of income polarization. The general form of MRP index does not allow for an assessment of the degree of economic polarization in the strict sense, i.e. the polarization that leads to the disappearance of the middle income class. However, the proposed MRP partial indices do allow for this type of analysis. The proposed indices allow also for the analysis of the polarization/convergence within subpopulations of interest. Moreover, the existing measures of polarization are focused only on comparing shapes of the incomes' distributions. The indices proposed in the study, paired with panel datasets, can be used to trace polarization experienced at individual level, and therefore assess polarization of incomes within subgroups which in fact have changed or maintained their income class belongingness. More generally, these tools can be used to assess income polarization in any given subpopulation such as socio-economic strata, ethnic minorities, people living in urban/rural areas, etc. We believe that the proposed approach forms a bridge between polarization and mobility measures, enabling researchers to conduct more thorough analysis within both frameworks.

The empirical analysis of changes in household income distribution was conducted for Poland in the 2005-2015 period. During these years the flows of households to the middle income class, resulting in income economic convergence, were higher than the outflows from this class, resulting in economic polarization. As a result, the middle income class has grown. However, the MRP index was nearly equal to zero, indicating virtually no income convergence in the whole population.

Analysis of the values of the proposed MRP partial indices, estimated for the distinguished income subpopulations, unveils a more intricate picture of relative income changes. Individuals departing from the middle income class, that is households experiencing economic polarization, have experienced, on average, a stronger shift in their relative incomes as compared to those who have entered middle income class, which are households experiencing economic convergence. The overall picture of lack of convergence in the whole population was caused by the fact that the stronger polarization in the first group was compensated by the higher number of households in the second group.

Moreover, households which belonged to the middle income class in the both analyzed years experienced a slight polarization of incomes, while households which stayed outside of the middle income class in both periods experienced a threefold stronger income convergence. If this trend continues, the fraction of the middle income class will further increase in the near future.

Open Access This article is licensed under a Creative Commons Attribution 4.0 International License, which permits use, sharing, adaptation, distribution and reproduction in any medium or format, as long as you give appropriate credit to the original author(s) and the source, provide a link to the Creative Commons licence, and indicate if changes were made. The images or other third party material in this article are included in the article's Creative Commons licence, unless indicated otherwise in a credit line to the material. If material is not included in the article's Creative Commons licence and your intended use is not permitted by statutory regulation or exceeds the permitted use, you will need to obtain permission directly from the copyright holder. To view a copy of this licence, visit http://creativecommons.org/licenses/by/4.0/. 


\section{References}

Anderson, G. (2004). Toward an empirical analysis of polarization. Journal of Econometrics, 122(1), 1-26.

Atkinson, A., \& Brandolini, A. (2011). On the identification of the middle class. Income Inequality. https:// doi.org/10.11126/stanford/9780804778244.003.0003.

Banerjee, A., \& Duflo, E. (2008). What is middle class about the middle classes around the world? Journal of Economic Perspectives, 22(2), 3-28.

Bossert, W., \& Schworm, W. (2008). Class of two-group polarization measures. Journal of Public Economic Theory, 10(6), 1169-1187.

Chakravarty, S. R., \& D’Ambrosio, C. (2010). Polarization orderings of income distributions. Review of Income and Wealth, 56(1), 47-64.

Czapiński, J., Kotowska, I. E., \& Panek, T. (2015). The research method. Social Diagnosis 2015. The objective and subjective quality of life in Poland. Contemporary Economics, 9(4), 24-33.

Duclos, J.-Y., Esteban, J., \& Ray, D. (2004). Polarization: Concepts, measurement, estimation. Econometrica, 72(6), 1737-1772.

Esteban, J., Gradin, C., \& Ray, D. (1998 as WP, 2007). Extension of a measure of polarization, with an application to the income distribution of five OECD countries. Journal of Economic Inequality, 5(1), $1-19$.

Esteban, J., \& Ray, D. (1991 as WP, 1994). On the measurement of polarization. Econometrica, 62(4), 819-852.

Esteban, J.-M., \& Ray, D. (2011). Linking conflict to inequality and polarization. The American Economic Review, 101, 1345-1374.

Foster, J., \& Wolfson, M. C. (1992 as WP, revised in 2009, published 2010). Polarization and the decline of the middle class: Canada and the US. Journal of Economic Inequality, 8(2), 247-273.

Handcock, M. S., \& Morris, M. (1999). Relative distribution methods in the social sciences. New York: Springer.

Horrigan, H., \& Haugen, S. (1988). The declining middle class: A sensitivity analysis. Monthly Labour Review, 111(5), 3-13.

Keefer, P., \& Knack, S. (2002). Polarization, politics and property rights: Links between inequality and growth. Public Choice, 111(1-2), 127-154.

Kharas, H., \& Gertz, G. (2010). The new global middle class: A cross-over from west to east. In C. Li (Ed.), China's emerging middle class: Beyond economic transformation. Washington, DC: Brookings Institution Press.

Kot, S. M. (2008). Economic polarization. Theory and application. Warsaw: Polish Scientific Publishers PWN. (in Polish).

Kuttner, B. (1983). The declining middle. The Atlantic Monthly, 108(1), 60-72.

Lasso de la Vega, M. C., Urrutia, A., \& Diez, H. (2010). Unit consistency and bipolarization of income distributions. Review of Income and Wealth, 56(1), 65-83.

Montalvo, J. G., \& Reynal-Querol, M. (2005). Ethnic polarization, Potential conflict and civil war. American Economic Review, 95(3), 796-816.

Morris, M., Bernhardt, A. D., \& Handcock, M. S. (1994). Economic inequality: New methods for new trends. American Sociological Review, 59(2), 205-219.

Panek, T. (2015). Methodology of analysing poverty. in: Czapiński, J., et al., Social Diagnosis 2015. The objective and subjective quality of life in Poland. Economics, 9(4), 510-517.

Panek, T. (2017). Economic polarization in Poland. Wiadomości Statystyczne, 1, 41-61. (in Polish).

Rodriguez, J. G., \& Salas, R. (2003). Extended bi-polarization and inequality measures. Research on Economic Inequality, 9, 69-83.

Rosenthal, N. (1985). The shrinking middle class: Myth or reality? Monthly Labour Review, 108(3), 3-19.

Shorrocks, A. (1978). Income inequality and income mobility. Journal of Economic Theory, 19(2), 376-393.

Szulc, A. (2003). It is possible to estimate reliable household equivalence scales. Statistics in Transition, 6(4), 589-611.

Thurow, L. (1984). The disappearance of the middle class (Vol. 133). New York: New York Times.

Vacas-Soriano, C., \& Fernandez-Macias, E. (2017). Income inequalities and employment patterns in Europe before and after the great recession. Eurofound, Luxembourg: Publications Office of the European Union. https://www.eurofound.europa.eu/publications/report/2017/income-inequalities-andemploym ent-patterns-in-europe-before-and-after-the-great-recession.

Wang, Y. Q., \& Tsui, K. Y. (2000). Polarization orderings and new classes of polarization indices. Journal of Public Economic Theory, 2, 349-363.

Wolfson, M. C. (1994). When inequalities diverge. American Economic Review, 84(2), 353-358. 
Wolfson, M. C. (1997). Divergent inequalities: Theory and empirical results. Review of Income and Wealth, 43(4), 401-421.

Publisher's Note Springer Nature remains neutral with regard to jurisdictional claims in published maps and institutional affiliations. 